\title{
Predicting the outcome of COVID-19 infection in kidney transplant recipients
}

Ozgur Akin Oto ${ }^{*}$, Savas Ozturk², Kenan Turgutalp ${ }^{3}$, Mustafa Arici ${ }^{4}$, Nadir Alpay ${ }^{5}$, Ozgur Merhametsiz ${ }^{6}$,

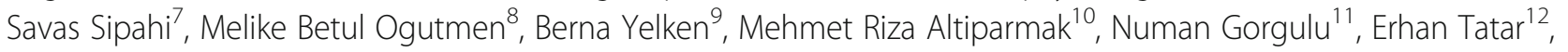
Oktay Ozkan², Yavuz Ayar ${ }^{13}$, Zeki Aydin ${ }^{14}$, Hamad Dheir ${ }^{15}$, Abdullah Ozkok ${ }^{16}$, Seda Safak', Mehmet Emin Demir ${ }^{6}$, Ali Riza Odabas ${ }^{17}$, Bulent Tokgoz ${ }^{18}$, Halil Zeki Tonbul ${ }^{19}$, Siren Sezer ${ }^{20}$, Kenan Ates ${ }^{21}$ and Alaattin Yildiz

\begin{abstract}
Background: We aimed to present the demographic characteristics, clinical presentation, and outcomes of our multicenter cohort of adult KTx recipients with COVID-19.

Methods: We conducted a multicenter, retrospective study using data of patients hospitalized for COVID-19 collected from 34 centers in Turkey. Demographic characteristics, clinical findings, laboratory parameters (hemogram, CRP, AST, ALT, LDH, and ferritin) at admission and follow-up, and treatment strategies were reviewed. Predictors of poor clinical outcomes were analyzed. The primary outcomes were in-hospital mortality and the need for ICU admission. The secondary outcome was composite in-hospital mortality and/or ICU admission.

Results: One hundred nine patients (male/female: 63/46, mean age: $48.4 \pm 12.4$ years) were included in the study. Acute kidney injury (AKI) developed in 46 (42.2\%) patients, and 4 (3.7\%) of the patients required renal replacement therapy (RRT). A total of $22(20.2 \%)$ patients were admitted in the ICU, and 19 (17.4\%) patients required invasive mechanical ventilation. 14 (12.8\%) of the patients died. Patients who were admitted in the ICU were significantly older (age over 60 years) (38.1\% vs 14.9\%, $p=0.016) .23$ (21.1\%) patients reached to composite outcome and these patients were significantly older (age over 60 years) $(39.1 \%$ vs. $13.9 \% ; p=0.004)$, and had lower serum albumin (3.4 $\mathrm{g} / \mathrm{dl}$ [2.9-3.8] vs. $3.8 \mathrm{~g} / \mathrm{dl}$ [3.5-4.1], $p=0.002)$, higher serum ferritin (679 $\mu \mathrm{g} / \mathrm{L}$ [184-2260] vs. $331 \mu \mathrm{g} / \mathrm{L}$ [128-839], $p=$

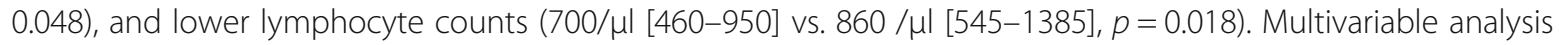
identified presence of ischemic heart disease and initial serum creatinine levels as independent risk factors for mortality, whereas age over 60 years and initial serum creatinine levels were independently associated with ICU admission. On analysis for predicting secondary outcome, age above 60 and initial lymphocyte count were found to be independent variables in multivariable analysis.
\end{abstract}

Conclusion: Over the age of 60 , ischemic heart disease, lymphopenia, poor graft function were independent risk factors for severe COVID-19 in this patient group. Whereas presence of ischemic heart disease and poor graft function were independently associated with mortality.

Keywords: Kidney transplantation, COVID-19, Registry

\footnotetext{
*Correspondence: maviozgurluk@gmail.com

'Division of Nephrology, Department of Internal Medicine, Istanbul University Istanbul Faculty of Medicine, Istanbul, Turkey

Full list of author information is available at the end of the article
}

\section{$\triangle B M C$}

C C The Author(s). 2021 Open Access This article is licensed under a Creative Commons Attribution 4.0 International License, which permits use, sharing, adaptation, distribution and reproduction in any medium or format, as long as you give appropriate credit to the original author(s) and the source, provide a link to the Creative Commons licence, and indicate if changes were made. The images or other third party material in this article are included in the article's Creative Commons licence, unless indicated otherwise in a credit line to the material. If material is not included in the article's Creative Commons licence and your intended use is not permitted by statutory regulation or exceeds the permitted use, you will need to obtain permission directly from the copyright holder. To view a copy of this licence, visit http://creativecommons.org/licenses/by/4.0/ The Creative Commons Public Domain Dedication waiver (http://creativecommons.org/publicdomain/zero/1.0/) applies to the data made available in this article, unless otherwise stated in a credit line to the data. 


\section{Introduction}

The novel coronavirus 2019 disease (COVID-19), which originated in the city of Wuhan, in Hubei province, China, infected more than 33 million people and caused nearly 1 million reported deaths worldwide (https:// coronavirus.jhu.edu/map.html). Studies addressing the risk factors, clinical features, and prognosis of the disease have been published [1,2]. Approximately $20 \%$ of COVID-19 patients have been reported to have moderate to severe clinical manifestations and 5\% progress to critical illness [3]. The case fatality rates vary in different reports. In general, it ranged from 1 to $7.2 \%$ and reached $49 \%$ among patients with critical illnesses $[3,4]$. The presence of comorbidities such as old age and diabetes mellitus, hypertension, chronic kidney disease, morbid obesity, coronary heart disease, and chronic lung disease have been identified as major risk factors for severe disease [5]. However, the diagnosis and clinical course of the disease in solid organ transplant (SOT) recipients may differ from the general population due to chronic immunosuppression and coexisting conditions [6]. There is scarce information on the infectious course of COVID-19 in transplant recipients. Although there are currently a couple of reports of COVID-19 among kidney transplant (KTx) recipients [7-9], it is yet unclear whether the presence of immunosuppression increases the complications of COVID-19 [10]. Previous reports suggest that immunosuppression may reduce the frequency of cytokine storms, a significant cause of mortality $[11,12]$. We aimed to present the clinical manifestations, course of the disease and outcomes of a large multicenter cohort of adult KTx recipients with COVID-19 in this study. We also examined the predictors of worse clinical outcomes in this group of patients.

\section{Methods}

\section{Study design and participants}

This multicenter, retrospective cohort study was conducted using data collected from 34 centers in Turkey under the unconditional support of the Turkish Society of Nephrology. One hundred nine patients (63 males, 46 females, mean age $48.4 \pm 12.4$ years old) were included between April 17 and June 1, 2020. The diagnosis of COVID-19 was based on the clinical symptoms, polymerase chain reaction (PCR) test for SARS-CoV-2 from the nasopharyngeal swab, and/or radiological findings. We also considered the patients whose first swab PCR test was negative, but the repeated test was positive, to be confirmed cases. Moreover, the patients whose clinical and radiological findings were consistent with COVID-19, but swab PCR tests were negative or not available, were also considered as "probable COVID-19 patients" and were included in this study [13]. The diagnosis was made with swab PCR positivity in 72 patients
(66.1\%). In 37 patients (33.9\%), swab PCR was negative. Laboratory tests (such as CRP, LDH, AST, and complete blood counts) of the patients were monitored daily during their hospitalization period (including ICU stay). It was analyzed to obtain target levels.

We excluded the patients who were pregnant, younger than 18 years of age, lack hospital discharge or survival data, were still hospitalized at the time of data collection, and the patients hospitalized for non-COVID-19 reasons from this study. The study was approved by the University of Health Sciences, Istanbul Haseki Training and Research Hospital Ethics Committee (number 41-2020). The selection of the study population is shown in Fig. 1.

\section{Data collection}

All participating centers scanned the electronic health records in hospital systems and recorded the data. We collected the admission data, including demographic information, duration of symptoms from onset to hospital admission, smoking habits, comorbidities and medications, initial serum creatinine, serum albumin, ferritin, C-reactive protein (CRP), hemoglobin, lymphocyte, and platelet counts. Dataset also included the clinical severity of COVID-19, laboratory tests performed during hospitalization, treatment for COVID-19 at the hospital, and the outcomes. COVID-19 severity was classified according to the suggestions in our national guideline [14]. The clinical severity of COVID-19 was defined according to the clinical presentation of COVID-19 at hospital admission and separated into four categories: patients with mild clinical symptoms without dyspnea or any sign of viral pneumonia on chest computerized tomography (CT) findings were defined as the mild disease, and patients with symptoms like fever and cough, dyspnea and signs of viral pneumonia on chest CT findings were as the moderate disease. The term severe disease referred to the patients requiring oxygen support on admission and the term critical disease referred to the patients who were hypoxic at admission and requiring close monitoring and/or need intensive care unit (ICU).

In terms of changes in immunosuppression, firstly, antimetabolite agents were discontinued. Calcineurin inhibitors (CNI) treatments were discontinued or the doses were reduced according to the severity of the disease in KTx patients (Tables 1, 2 and 3). No changes were made in immunosuppressive treatments in patients that were considered as "mild case" at admission ( $\mathrm{n}: 3$, $3.8 \%$ ). However, MPA/AZA was discontinued in mild cases whose clinical condition did not improve within 3-5 days or in patients considered to be "moderate case" at the time of admission (n: 92, 84.4\%). In patients with severe/critical COVID-19, all immunosuppressive drugs except steroids were discontinued (n: 14, 12.8\%). All modifications were made by the investigator's initiative. 


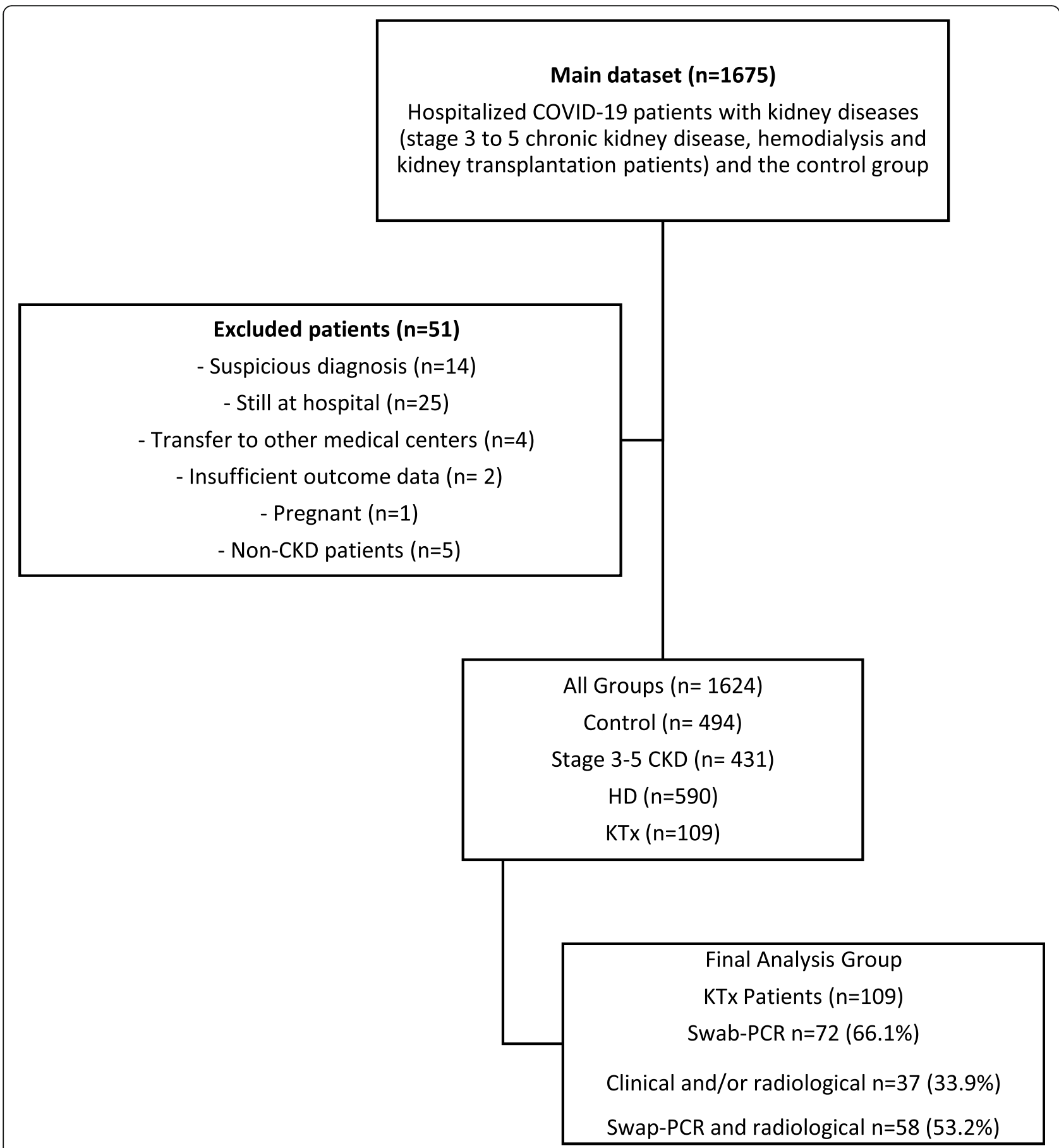

Fig. 1 Flow chart illustrating the selection of the study population. Abbreviations: CKD: chronic kidney disease, HD: hemodialysis, KTx: kidney transplantation, PCR: polymerase chain reaction

\section{Outcomes}

The primary outcomes were in-hospital mortality and the need for ICU admission. The secondary outcome was composite in-hospital mortality and/or ICU admission. Length of stay (LOS) at the hospital was used in the in-hospital mortality analyses, which was defined as the period starting from the day of hospitalization and ending on the day of death, admission to the ICU, or discharge. Acute kidney injury (AKI) was defined by the following criteria determined by KDIGO guidelines: increase in serum creatinine $\geq 0.3 \mathrm{mg} / \mathrm{dl}$ or increase in serum creatinine to $>1.5$ times the baseline creatinine levels [15]. The need for renal replacement therapy (RRT) and the requirement of invasive mechanical 
Table 1 Baseline characteristics, lab tests, medication, and follow-up parameters of the patients according to survival

\begin{tabular}{|c|c|c|c|c|c|}
\hline \multicolumn{2}{|l|}{ Characteristic } & $\begin{array}{l}\text { All patients } \\
N=109\end{array}$ & $\begin{array}{l}\text { Survivors } \\
(N=95)\end{array}$ & $\begin{array}{l}\text { Non- } \\
\text { Survivors } \\
(N=14)\end{array}$ & $p$-value \\
\hline \multicolumn{2}{|l|}{ Gender, Male, n (\%) } & $63(57.8)$ & $57(60.0)$ & $6(42.9)$ & 0.225 \\
\hline \multicolumn{2}{|l|}{ Age (years), mean $\pm S D$} & $48.4 \pm 12.4$ & $47.9 \pm 12.4$ & $51.7 \pm 12.4$ & 0.334 \\
\hline \multirow[t]{2}{*}{ Donor type, n, (\%) } & Deceased & $17(15.6)$ & $14(14.7)$ & $3(21.4)$ & 0.519 \\
\hline & Living & $92(84.4)$ & $81(85.3)$ & $11(78.6)$ & \\
\hline \multicolumn{2}{|l|}{ Age > 60 years, $\mathrm{n}(\%)$} & $21(19.4)$ & $16(76.2)$ & $5(23.8)$ & 0.065 \\
\hline \multicolumn{2}{|c|}{ Time from symptom onset to admission, days, median (IQR) } & $4.5(3.0-7.0)$ & $5.0(3.0-7.0)$ & $4.0(3.0-8.5)$ & 0.634 \\
\hline \multicolumn{2}{|l|}{ LOS (days), median (IQR) } & $9.0(6.0-14.0)$ & $8.0(6.0-13.5)$ & $14(8.0-17.5)$ & 0.038 \\
\hline \multicolumn{2}{|c|}{ Time to from symptom onset to death or discharge, days, median (IQR) } & $\begin{array}{l}14.0(10.0- \\
21.0)\end{array}$ & $\begin{array}{l}13.0(10.0- \\
20.0)\end{array}$ & $\begin{array}{l}21.0(13.0- \\
25.0)\end{array}$ & 0.092 \\
\hline \multicolumn{2}{|l|}{ Tx Duration, years, median (IQR) } & $5.0(3.0-9.0)$ & $5.0(2.9-9.0)$ & $5(3.0-8.0)$ & 0.899 \\
\hline \multicolumn{2}{|l|}{ Tx Duration < 1 year $n(\%)$} & $17(15.6)$ & $15(15.8)$ & $2(14.3)$ & 0.885 \\
\hline \multirow[t]{7}{*}{ Coexisting disorder, n/N (\%) } & Diabetes mellitus & 25/107 (23.4) & 21/93 (22.6) & $4 / 14(28.6)$ & 0.621 \\
\hline & Hypertension & $81 / 106(76.4)$ & $70 / 92(76.1)$ & $11 / 14(78.6)$ & 0.838 \\
\hline & Ischemic heart disease & 18/103 (17.5) & 13/90 (15.3) & $5 / 13(38.5)$ & 0.033 \\
\hline & Heart failure & $4 / 105(3.8)$ & 2/91 (2.2) & 2/14 (14.3) & 0.033 \\
\hline & COPD & $5 / 105(4.8)$ & 4/92 (4.6) & $1 / 13(7.7)$ & 0.491 \\
\hline & Cancer & $6 / 105(5.7)$ & 6/92 (6.9) & 0/13 (0) & 1.000 \\
\hline & Chronic liver disease & $1 / 105(1.0)$ & $1 / 92(1.1)$ & $0(0)$ & 1.000 \\
\hline \multirow[t]{9}{*}{ Cause of kidney disease, n (\%) } & Diabetic nephropathy & $13(11.9)$ & $10(10.5)$ & $3(21.4)$ & 0.764 \\
\hline & Glomerular disease & $13(11.9)$ & $9(9.4)$ & $4(28.6)$ & \\
\hline & Hypertensive nephrosclerosis & $28(25.7)$ & $25(26.3)$ & $3(21.4)$ & \\
\hline & ADPCKD & $5(4.6)$ & $5(5.2)$ & $0(0)$ & \\
\hline & Amyloidosis & $4(3.7)$ & $4(4.2)$ & $0(0)$ & \\
\hline & Chronic pyelonephritis & $6(5.5)$ & $6(6.3)$ & $0(0)$ & \\
\hline & Urological diseases & $6(5.5)$ & $6(6.3)$ & $0(0)$ & \\
\hline & Unknown & $31(28.4)$ & $27(28.4)$ & $4(28.5)$ & \\
\hline & Others & $3(2.7)$ & $3(3.1)$ & $0(0)$ & \\
\hline \multirow[t]{4}{*}{ Smoking, n (\%) } & Former smoked & $22(20.2)$ & $20 / 90(22.2)$ & 2/14 (14.3) & 0.834 \\
\hline & Never smoker & $43(39.4)$ & $36 / 90(40.0)$ & $7 / 14(50.0)$ & \\
\hline & Current smoker & $1(0.9)$ & $1 / 90(1.1)$ & 0/14 (0) & \\
\hline & Unknown & $43(39.4)$ & $38 / 90(42.2)$ & $5 / 14(35.7)$ & \\
\hline \multirow[t]{11}{*}{ Medications, n/N (\%) } & ACEi & 21/103 (20.4) & 17/85 (20.0) & 4/14 (28.6) & 0.414 \\
\hline & ARBs & $14 / 102(13.7)$ & $14 / 87(16.1)$ & $0 / 14(0)$ & 0.108 \\
\hline & Statins & 11/101 (10.9) & $9(9.5)$ & $2(14.0)$ & 0.308 \\
\hline & $\begin{array}{l}\text { Anticoagulant or antiplatelet } \\
\text { agent }\end{array}$ & 45/102 (44.1) & $37(41.1)$ & $8(66.7)$ & 0.094 \\
\hline & Oral antidiabetics & 8/102 (7.8) & $7(8.0)$ & $1(7.4)$ & 0.916 \\
\hline & Tacrolimus & 86/109 (78.9) & 75 (78.9) & 11 (78.6) & 0.974 \\
\hline & CsA & 9/109 (8.3) & $9(9.5)$ & $0(0)$ & 0.229 \\
\hline & MPA derivatives & $94 / 109(86.2)$ & $80(84.2)$ & $14(100)$ & 0.109 \\
\hline & MTORi & 12/109 (11.0) & $10(10.5)$ & $2(14.3)$ & 0.651 \\
\hline & Azathioprine & $6 / 103(5.5)$ & $6(6.3)$ & $0(0)$ & 0.333 \\
\hline & Prednisone & 106/109 (97.2) & $92(96.8)$ & $14(100)$ & 1.000 \\
\hline
\end{tabular}


Table 1 Baseline characteristics, lab tests, medication, and follow-up parameters of the patients according to survival (Continued)

\begin{tabular}{|c|c|c|c|c|c|}
\hline Characteristic & & $\begin{array}{l}\text { All patients } \\
N=109\end{array}$ & $\begin{array}{l}\text { Survivors } \\
(N=95)\end{array}$ & $\begin{array}{l}\text { Non- } \\
\text { Survivors } \\
(N=14)\end{array}$ & $p$-value \\
\hline Induction therapy, yes, n, (\%) & & $80(73.4)$ & $71(74.7)$ & $9(64.3)$ & 0.409 \\
\hline \multirow[t]{2}{*}{ Induction therapy n, (\%) } & ATLG & $67(61.5)$ & $61(64.2)$ & $6(42.9)$ & \multirow[t]{2}{*}{0.270} \\
\hline & Basiliximab & $13(11.9)$ & $10(10.5)$ & $3(21.4)$ & \\
\hline \multirow[t]{4}{*}{ Modification of immunosuppression, n, (\%) } & No changed & $3(2.8)$ & $3(3.2)$ & $0(0.0)$ & \multirow[t]{4}{*}{0.163} \\
\hline & CNI withdrawal & $0(0.0)$ & $0(0.0)$ & $0(0.0)$ & \\
\hline & MPA/AZA withdrawal & $92(84.4)$ & $82(86.3)$ & $10(71.4)$ & \\
\hline & CNI + MPA/AZA withdrawal & $14(12.8)$ & $10(10.5)$ & $4(28.6)$ & \\
\hline \multirow{3}{*}{$\begin{array}{l}\text { COVID-19 related clinic presentation at the time of } \\
\text { diagnosis, } \mathrm{n}(\%)\end{array}$} & Mild disease & $67(61.4)$ & $65(59.6)$ & $2(14.3)$ & \multirow[t]{3}{*}{$<0.001$} \\
\hline & Moderate Disease & $33(30.3)$ & $27(28.4)$ & $6(42.9)$ & \\
\hline & Severe-Critical Disease & $9(8.3)$ & $3(3.2)$ & $6(42.9)$ & \\
\hline \multirow[t]{8}{*}{ Presentation symptoms n, (\%) } & Fever & $70(64.2)$ & $60(63.2)$ & $10(71.4)$ & 0.547 \\
\hline & Myalgia & $32(29.4)$ & $29(30.5)$ & $3(21.4)$ & 0.485 \\
\hline & Dyspnea & $53(48.6)$ & $41(43.2)$ & $12(85.7)$ & 0.003 \\
\hline & Diarrhea & $12(11.0)$ & $11(11.6)$ & $1(7.1)$ & 0.621 \\
\hline & Cough & $72(66.1)$ & $64(67.4)$ & $8(57.1)$ & 0.451 \\
\hline & Throat pain & $6(5.5)$ & $5(5.3)$ & $1(7.1)$ & 0.773 \\
\hline & Headache & $14(12.8)$ & $14(14.7)$ & $0(0.0)$ & 0.124 \\
\hline & Fatigue & $47(43.1)$ & $38(40.0)$ & $9(64.3)$ & 0.504 \\
\hline \multirow[t]{9}{*}{ COVID-19 drug treatments, n/N (\%) } & Macrolides & $71 / 106(67.0)$ & $60(64.5)$ & $11(84.6)$ & 0.149 \\
\hline & Oseltamivir & $59 / 105(56.2)$ & $50(54.3)$ & $9(69.2)$ & 0.311 \\
\hline & Hydroxychloroquine & 108/109 (99.1) & $94(98.9)$ & $14(100.0)$ & 0.700 \\
\hline & Lopinavir-ritonavir & 10/94 (10.6) & $10(12.2)$ & $0(.0)$ & 0.201 \\
\hline & Favipiravir & $49 / 100(49.0)$ & $38(43.2)$ & $11(91.7)$ & 0.002 \\
\hline & Glucocorticoids & $59 / 101(58.4)$ & $47(54.0)$ & $12(85.7)$ & 0.026 \\
\hline & Tocilizumab & $10 / 99(10.1)$ & $5(5.7)$ & $5(41.7)$ & $<0.001$ \\
\hline & Anakinra & $3 / 100(3.0)$ & $3(3.4)$ & $O(.0)$ & 0.497 \\
\hline & $\begin{array}{l}\text { Apheresis / } \\
\text { immunoadsorption }\end{array}$ & $3 / 100(3.0)$ & $2(2.3)$ & $1(7.7)$ & 0.357 \\
\hline \multirow[t]{7}{*}{ Laboratory findings at admission, median (IQR) } & Creatinine $(\mu \mathrm{mol} / \mathrm{l})$ & $\begin{array}{l}132.6(97.2- \\
194.5)\end{array}$ & $\begin{array}{l}132.6(97.2- \\
181.2)\end{array}$ & $\begin{array}{l}198.0(97.2- \\
293.7)\end{array}$ & 0.018 \\
\hline & Albumin $(g / d l)$ & $3.8(3.4-4.1)$ & $3.8(3.4-4.0)$ & $3.5(3.0-3.83)$ & 0.170 \\
\hline & Ferritin $(\mu \mathrm{g} / \mathrm{l})$ & $\begin{array}{l}369.5(152- \\
906)\end{array}$ & $\begin{array}{l}338.0(132- \\
891)\end{array}$ & $\begin{array}{l}679(265- \\
1718)\end{array}$ & 0.112 \\
\hline & Hemoglobin $(\mathrm{g} / \mathrm{dl})$ mean $\pm \mathrm{SD}$ & $11.6 \pm 2.3$ & $11.5 \pm 2.4$ & $12.0 \pm 1.9$ & 0.195 \\
\hline & Lymphocyte count $(/ \mu l)$ & $\begin{array}{l}850(541- \\
1257)\end{array}$ & $\begin{array}{l}850(540- \\
1330)\end{array}$ & 790(557-1015) & 0.412 \\
\hline & $\begin{array}{l}\text { Lymphopenia }(<800 / \mu \mathrm{L}) \mathrm{n} \text {, } \\
(\%)\end{array}$ & $53(48.6)$ & $46(48.4)$ & $7(50.0)$ & 0.912 \\
\hline & Platelet count $\left(\times 10^{3} / \mu \mathrm{l}\right)$ & $199(171-245)$ & $199(170-248)$ & $189(158-239)$ & 0.474 \\
\hline \multirow[t]{5}{*}{ Follow-up parameters } & ICU admission, $\mathrm{n}(\%)$ & $22(20.2)$ & $9(40.9)$ & $13(59.1)$ & $<0.001$ \\
\hline & Bacterial superinfection, n, (\%) & $9(8.3)$ & $7(7.4)$ & $2(14.3)$ & 0.416 \\
\hline & $\begin{array}{l}\text { Mechanical ventilation in ICU, } \\
\mathrm{n}(\%)\end{array}$ & $19(17.4)$ & $6(31.6)$ & $13(68.4)$ & 0.025 \\
\hline & Acute kidney injury, n (\%) & $46(42.2)$ & $36(37.9)$ & $10(71.4)$ & 0.018 \\
\hline & RRT, n (\%) & $4(3.7)$ & $1(1.1)$ & $3(21.4)$ & $<0.001$ \\
\hline
\end{tabular}


Table 1 Baseline characteristics, lab tests, medication, and follow-up parameters of the patients according to survival (Continued)

\begin{tabular}{|c|c|c|c|c|c|}
\hline \multicolumn{2}{|l|}{ Characteristic } & \multirow{2}{*}{$\begin{array}{l}\text { All patients } \\
N=109 \\
36(33.0)\end{array}$} & \multirow{2}{*}{$\begin{array}{l}\text { Survivors } \\
(\boldsymbol{N}=\mathbf{9 5})\end{array}$} & \multirow{2}{*}{$\begin{array}{l}\text { Non- } \\
\text { Survivors } \\
(\boldsymbol{N}=14) \\
3(21.4)\end{array}$} & \multirow{2}{*}{$\begin{array}{l}\boldsymbol{p} \text {-value } \\
0.323\end{array}$} \\
\hline & Leukopenia $\left(<4.0 \times 10^{3} / \mu l\right)$ & & & & \\
\hline & Lymphopenia $(<800 / \mu l)$ & 77/108 (71.3) & $66(69.5)$ & $11(84.6)$ & 0.258 \\
\hline & $\begin{array}{l}\text { Thrombocytopenia }(<150 \times \\
\left.10^{3} / \mu \mathrm{L}\right)\end{array}$ & $16(14.7)$ & $13(13.7)$ & $3(21.4)$ & 0.445 \\
\hline & $\begin{array}{l}\mathrm{LDH}(>2 \times \text { upper limit of } \\
\text { normal) }\end{array}$ & 29/104 (27.9) & $21(23.1)$ & $8(61.5)$ & 0.004 \\
\hline & $\begin{array}{l}\text { AST ( }>2 \times \text { upper limit of } \\
\text { normal) }\end{array}$ & 15/98 (15.3) & $10(11.8)$ & $5(38.5)$ & 0.013 \\
\hline & $\begin{array}{l}\text { CRP (> } 10 \times \text { upper limit of } \\
\text { normal) }\end{array}$ & $47(43.1)$ & 37 (38.9) & $10(71.4)$ & 0.022 \\
\hline
\end{tabular}

$p$-values presented from the chi-square test, Fisher's exact test, t-test, or Mann-Whitney $\mathrm{U}$ test

Abbreviations: IQR interquartile range, LOS length of stay in the hospital, COPD chronic obstructive pulmonary disease, ADPCKD autosomal dominant polycystic kidney disease, CSA cyclosporine A, ACEi angiotensin-converting enzyme inhibitors, ARBs angiotensin II receptor blocker, mTORi mammalian target of rapamycin inhibitors, MPA mycophenolate derivatives, CNI calcineurin inhibitors, AZA azathioprine, ATLG anti-T lymphocyte globulin, RRT renal replacement therapy, CRP C reactive protein, $L D H$ lactate dehydrogenase, $A S T$ aspartate aminotransferase, $I C U$ intensive care unit

ventilation (IMV) for patients admitted in the ICU were recorded.

\section{Statistical analysis}

The analyses were performed using the IBM SPSS Statistics for Windows, Version 23.0 (IBM Corp., Armonk, NY, USA). We summarized descriptive statistics as numbers and percentages for categorical variables, and mean, standard deviation, median, minimum-maximum, and interquartile range (IQR) for numerical variables, where appropriate. For the comparisons of categorical variables, the chi-square test or Fisher's exact test (when expected frequencies for some cells are $<5$ ) were used. We used Student's t-test to compare the two independent groups in the analyses of the normally distributed numerical data, and the Mann-Whitney-U test in the case of abnormal distribution of numerical data. To find out the independent parameters related to primary and secondary outcomes, we created a logistic regression analysis model with the entering method using parameters that included demographic, clinical, and laboratory parameters that suggested a potential effect on the outcomes in univariate analyses. Parameters with $p<0.05$ in univariate analyzes were considered significant and they were included in multivariate analyzes. A $P$-value of $<$ 0.05 was considered significant.

\section{Results}

\section{Demographic and clinical characteristics}

A total of 109 KTx recipients hospitalized with COVID19 from 34 different centers were included in the study. $63(57.8 \%)$ were male, and the mean age was $48.4 \pm 12.4$ (19.4\% more than 60 ) years (Table 1 ). Hypertension was the most common coexisting disorder affecting $76.4 \%$ of patients, followed by diabetes mellitus (23.4\%), ischemic heart disease (17.5\%), cancer (5.7\%), and chronic obstructive pulmonary disease (COPD) (4.8\%). $21.1 \%$ of the patients had a previous or current smoking history. The median time between transplantation and the diagnosis of COVID-19 was 5.0 (IQR 3.0-9.0) years. Table 1 shows the baseline characteristics of patients according to survival.

\section{Clinical outcome}

Median LOS was 9 days (IQR: 6-14 days). AKI developed in $46(42.2 \%)$, and $4(3.7 \%)$ patients needed RRT. A total of $22(20.2 \%)$ patients were admitted to ICU, and 19 (17.4\%) patients required IMV. The development of AKI (71.4\% vs. $37.9 \%$ respectively; $p=0.018)$, requiring IMV ( $68.4 \%$ vs. $31.6 \%$ respectively; $p=0.025)$, and need for RRT $(21.4 \%$ vs. $1.1 \%$, respectively; $p<0.001)$ were significantly higher in non-survivors compared to survivors. A total of 14 (12.8\%) patients died. 23 (21.1\%) patients reached the secondary outcome.

Parameters found to be associated with primary outcome and secondary outcome.

Ischemic heart disease and heart failure were higher in patients who died than surviving patients $(38.5 \%$ vs. $15.3 \%, p=0.033 ; 14.3 \%$ vs. $2.2 \%, p=0.028$, respectively) and those reaching secondary outcome $(31.8 \%$ vs $13.6 \%$, $p=0.046 ; 13.0 \%$ vs $1.2 \%, p=0.009$, respectively) (Tables $1,3)$. Non-survivor patients had longer LOS than other patients [14 days (IQR: $8-17.5$ days) vs. 8 days (IQR: $6-$ 13.5 days), $p=0.038$ ] (Table 1 ). ICU needs were observed significantly more frequently in patients with heart failure compared to other patients (Table 2).

Neither age, gender, transplantation duration, primary kidney disease, comorbidities (except as mentioned 
Table 2 Baseline characteristics, lab tests, medication, and follow-up parameters of the patients according to ICU admission

\begin{tabular}{|c|c|c|c|c|}
\hline \multirow{2}{*}{ Characteristic } & & \multicolumn{2}{|l|}{ ICU admission } & \multirow[t]{2}{*}{$p$-value } \\
\hline & & $\begin{array}{l}\text { No } \\
N=87\end{array}$ & $\begin{array}{l}\text { Yes } \\
N=22\end{array}$ & \\
\hline \multicolumn{5}{|l|}{ Demographic information } \\
\hline Male Gender, n (\%) & & $52(59.8)$ & $11(50.0)$ & 0.407 \\
\hline Age (years), median (IQR) & & $48(38.0-56.0)$ & $51(44.0-64.0)$ & 0.227 \\
\hline \multirow[t]{2}{*}{ Donor type, n, (\%) } & Deceased & $11(12.6)$ & $6(27.3)$ & 0.091 \\
\hline & Living & $76(87.4)$ & $16(72.7)$ & \\
\hline$>60$ years $n, \%$ & & $13(14.9)$ & $8(38.1)$ & 0.016 \\
\hline Time from symptom onset to admission, days, median (IQR) & & $4(3.0-7.0)$ & $5(3.0-7.0)$ & 0.536 \\
\hline Transplantation duration, years, median (IQR) & & $5(3.0-9.0)$ & $6(3.0-9.0)$ & 0.774 \\
\hline Length of stay in hospital (days), median (IQR) & & $9(6.0-13.0)$ & $14.5(8.0-18.0)$ & 0.003 \\
\hline Tx Duration < 1 year $\mathrm{n}(\%)$ & & $14(16.1)$ & $3(13.6)$ & 0.777 \\
\hline \multirow[t]{7}{*}{ Coexisting disorder, n/N (\%) } & Diabetes mellitus & $18 / 85(21.2)$ & $7 / 22(31.8)$ & 0.293 \\
\hline & Hypertension & 62/84 (73.8) & 19/22 (86.4) & 0.217 \\
\hline & Ischemic heart disease & $12 / 82(14.6)$ & 6/21 (28.6) & 0.133 \\
\hline & Heart failure & $1 / 83(1.2)$ & $3 / 22(13.6)$ & 0.007 \\
\hline & COPD & $3 / 84(3.6)$ & $2 / 21(9.5)$ & 0.252 \\
\hline & Cancer & $6 / 84(7.1)$ & $0 / 21(0.0)$ & 0.207 \\
\hline & Chronic liver disease & 4/84 (4.6) & $1 / 21(4.5)$ & 0.615 \\
\hline \multirow[t]{9}{*}{ Cause of kidney disease, n (\%) } & Diabetic nephropathy & $4(4.6)$ & $0(0.0)$ & 0.189 \\
\hline & Glomerular disease & $6(6.9)$ & $0(0.0)$ & \\
\hline & Hypertensive nephrosclerosis & $9(10.3)$ & $4(18.2)$ & \\
\hline & ADPCKD & $8(9.2)$ & $5(22.7)$ & \\
\hline & Amyloidosis & $22(25.3)$ & $6(27.3)$ & \\
\hline & Chronic pyelonephritis & $0(0.0)$ & $1(4.5)$ & \\
\hline & Urological diseases & $2(2.3)$ & $0(0.0)$ & \\
\hline & Unknown & $26(29.9)$ & $5(22.7)$ & \\
\hline & Others & $6(6.9)$ & $0(0.0)$ & \\
\hline \multirow[t]{4}{*}{ Smoking, n (\%) } & Former smoked & $18(20.7)$ & $4(18.2)$ & 0.890 \\
\hline & Never smoker & $33(37.9)$ & $10(45.5)$ & \\
\hline & Current smoker & $1(1.1)$ & $0(0.0)$ & \\
\hline & Unknown & $35(40.2)$ & $8(36.4)$ & \\
\hline \multirow[t]{11}{*}{ Medications, n/N (\%) } & ACEi & 17/82 (20.7) & $4 / 21(19.0)$ & 0.864 \\
\hline & ARBs & 12/81 (14.8) & $2 / 21(9.5)$ & 0.530 \\
\hline & Statins & $7 / 82(8.5)$ & $4 / 19(21.1)$ & 0.115 \\
\hline & $\begin{array}{l}\text { Anticoagulant or antiplatelet } \\
\text { agent }\end{array}$ & $34 / 83(41.0)$ & $11 / 19(57.9)$ & 0.180 \\
\hline & Oral antidiabetics & 7/81 (8.6) & $1 / 21(4.8)$ & 0.556 \\
\hline & Tacrolimus & $70(80.5)$ & $16(72.7)$ & 0.427 \\
\hline & CsA & $9(10.3)$ & $0(0.0)$ & 0.115 \\
\hline & MPA derivatives & $75(86.2)$ & 19 (86.4) & 0.985 \\
\hline & MTORi & $9(10.3)$ & $3(13.6)$ & 0.659 \\
\hline & Azathioprine & $5(5.7)$ & $1(4.5)$ & 0.825 \\
\hline & Prednisone & 85 (97.7) & $21(95.5)$ & 0.565 \\
\hline Induction, yes, n, (\%) & & $67(77.0)$ & $13(52.1)$ & 0.089 \\
\hline
\end{tabular}


Table 2 Baseline characteristics, lab tests, medication, and follow-up parameters of the patients according to ICU admission (Continued)

\begin{tabular}{|c|c|c|c|c|}
\hline \multirow{2}{*}{ Characteristic } & & \multicolumn{2}{|l|}{ ICU admission } & \multirow[t]{2}{*}{$p$-value } \\
\hline & & $\begin{array}{l}\text { No } \\
N=87\end{array}$ & $\begin{array}{l}\text { Yes } \\
N=22\end{array}$ & \\
\hline \multirow[t]{2}{*}{ Induction therapy n, (\%) } & ATLG & $57(65.5)$ & $10(45.5)$ & \multirow[t]{2}{*}{0.189} \\
\hline & Basiliximab & $10(11.5)$ & $3(13.6)$ & \\
\hline \multirow[t]{3}{*}{ Modification of immunosuppression n, (\%) } & No changed & $3(2.8)$ & $0(0.0)$ & \multirow[t]{3}{*}{0.059} \\
\hline & MPA/AZA withdrawal & $76(87.4)$ & $16(72.7)$ & \\
\hline & $\mathrm{CNI}+\mathrm{MPA} / \mathrm{AZA}$ withdrawal & $8(9.2)$ & $6(27.3)$ & \\
\hline \multirow{3}{*}{$\begin{array}{l}\text { COVID-19 related clinic presentation at the time of diagnosis, } n \\
\text { (\%) }\end{array}$} & Mild disease & $64(73.5)$ & $3(13.6)$ & \multirow[t]{3}{*}{$<0.001$} \\
\hline & Moderate Disease & $22(23.5)$ & $11(50.0)$ & \\
\hline & Severe-Critical Disease & $1(1.1)$ & $8(36.4)$ & \\
\hline \multirow[t]{8}{*}{ Presentation symptoms n, (\%) } & Fever & $55(63.2)$ & $15(68.2)$ & 0.664 \\
\hline & Myalgia & $27(31.0)$ & $5(22.7)$ & 0.445 \\
\hline & Dyspnea & $36(41.4)$ & $17(77.3)$ & 0.003 \\
\hline & Diarrhea & $11(12.6)$ & $1(4.5)$ & 0.278 \\
\hline & Cough & $60(69.0)$ & $12(54.5)$ & 0.202 \\
\hline & Throat pain & $4(4.6)$ & $2(9.1)$ & 0.409 \\
\hline & Headache & $11(12.6)$ & $3(13.6)$ & 0.901 \\
\hline & Fatigue & $36(41.4)$ & $11(50.0)$ & 0.466 \\
\hline \multirow[t]{9}{*}{ COVID-19 drug treatments, n/N (\%) } & Macrolides & $55(64.7)$ & $16(76.2)$ & 0.316 \\
\hline & Oseltamivir & $45(53.6)$ & $14(66.7)$ & 0.279 \\
\hline & Hydroxychloroquine & $86(98.9)$ & $22(100.0)$ & 0.613 \\
\hline & Lopinavir-ritonavir & $8(10.4)$ & $2(11.8)$ & 0.868 \\
\hline & Favipiravir & $31(38.3)$ & $18(94.7)$ & $<0.001$ \\
\hline & Glucocorticoids & $41(51.3)$ & $18(85.7)$ & 0.004 \\
\hline & Tocilizumab & $5(6.3)$ & $5(26.3)$ & 0.009 \\
\hline & Anakinra & $3(3.8)$ & $0(0.0)$ & 0.379 \\
\hline & Apheresis / immunoadsorption & $0(0.0)$ & $3(15.0)$ & $<0.001$ \\
\hline \multirow[t]{7}{*}{ Laboratory findings at admission, median (IQR) } & Creatinine $(\mu \mathrm{mol} / \mathrm{l})$ & $132.6(88.4-176.8)$ & $\begin{array}{l}198.0(106.1- \\
265.2)\end{array}$ & 0.016 \\
\hline & Albumin $(\mathrm{g} / \mathrm{dl})$ & $3.8(3.5-4.1)$ & $3.45(2.9-3.8)$ & 0.003 \\
\hline & Ferritin $(\mu \mathrm{g} / \mathrm{l})$ & 328 (129.0-814.0) & $\begin{array}{l}728(514.0- \\
2000.0)\end{array}$ & 0.029 \\
\hline & Hemoglobin $(\mathrm{g} / \mathrm{dl})$ mean \pm SD & $11.6(10.0-13.3)$ & $11.4(9.7-13.5)$ & 0.970 \\
\hline & Lymphocyte count $(/ \mu l)$ & $\begin{array}{l}860(547.0- \\
1380.0)\end{array}$ & $705(460.0-950.0)$ & 0.086 \\
\hline & 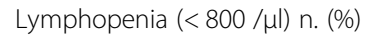 & $41(47.1)$ & $12(54.5)$ & 0.534 \\
\hline & Platelet count $\left(\times 10^{3} / \mu \mathrm{l}\right)$ & $200(170.0-249.0)$ & $185(161.0-232.0)$ & 0.275 \\
\hline \multirow[t]{7}{*}{ Follow-up parameters, n (\%) } & Acute kidney injury, n (\%) & $31(35.6)$ & $15(68.2)$ & 0.006 \\
\hline & RRT, n (\%) & $0(0.0)$ & $4(18.2)$ & $<0.001$ \\
\hline & Bacterial superinfection. N (\%) & $6(6.9)$ & $3(13.6)$ & 0.527 \\
\hline & \multicolumn{4}{|c|}{ Laboratory tests during hospitalization, n (\%) } \\
\hline & Leucopenia $(<4.0 / \mu l)$ & $28(32.2)$ & $8(36.4)$ & 0.710 \\
\hline & Lymphopenia $(800 / \mu l)$ & $57(66.3)$ & $20(90.9)$ & 0.023 \\
\hline & $\begin{array}{l}\text { Thrombocytopenia }\left(<150 \times 10^{3} /\right. \\
\mu l)\end{array}$ & $11(12.6)$ & $5(22.7)$ & 0.232 \\
\hline
\end{tabular}


Table 2 Baseline characteristics, lab tests, medication, and follow-up parameters of the patients according to ICU admission (Continued)

\begin{tabular}{|c|c|c|c|c|}
\hline \multirow{2}{*}{ Characteristic } & & \multicolumn{2}{|c|}{ ICU admission } & \multirow[t]{2}{*}{$p$-value } \\
\hline & & $\begin{array}{l}\text { No } \\
N=87\end{array}$ & $\begin{array}{l}\text { Yes } \\
N=22\end{array}$ & \\
\hline & $\mathrm{LDH}$ (> $2 \times$ upper limit of normal) & $13(15.7)$ & $16(76.2)$ & $<0.001$ \\
\hline & AST ( $>2 \times$ upper limit of normal) & $7(9.1)$ & $8(38.1)$ & 0.001 \\
\hline & CRP (> $10 \times$ upper limit of normal) & $31(35.6)$ & $16(72.7)$ & 0.002 \\
\hline \multirow[t]{2}{*}{ The final situation, n (\%) } & Recover & $86(98.9)$ & $9(40.9$ & $<0.001$ \\
\hline & Exitus & $1(1.1)$ & $13(59.1)$ & \\
\hline
\end{tabular}

$p$-values presented from the chi-square test, Fisher's exact test, t-test, or Mann-Whitney $\mathrm{U}$ test

Abbreviations: IQR interquartile range, $L O S$ length of stay in the hospital, COPD chronic obstructive pulmonary disease, $A D P C K D$ autosomal dominant polycystic kidney disease, CSA cyclosporine A, ACEi angiotensin-converting enzyme inhibitors, ARBs angiotensin II receptor blocker, mTORi mammalian target of rapamycin inhibitors, MPA mycophenolate derivatives, CNI calcineurin inhibitors, AZA azathioprine, ATLG anti-T lymphocyte globulin, RRT renal replacement therapy, CRP C reactive protein, $L D H$ lactate dehydrogenase, $A S T$ aspartate aminotransferase, $I C U$ intensive care unit

above), smoking history, maintenance immunosuppression nor use of angiotensin-converting enzyme inhibitors or angiotensin receptor blockers (ACEi/ARBs) was significantly different between the patients reaching primary and secondary outcomes (Tables 1,2,3).

Patients reaching the secondary outcome had longer LOS than other patients (14 days [IQR: $8-18.5$ days] vs. 8.5 days [IQR: $6-13$ days], $p<0.001$ ) (Table 3 ).

\section{Presentation, laboratory results, and treatment according to primary and secondary outcomes}

The most common symptoms at admission were coughing (66.1\%) and fever (64.2\%), followed by.

dyspnea $(48.6 \%)$ and fatigue $(43.1 \%)$. The presence of dyspnea $(85.7 \%$ vs. $43.2 \%, p=0.003)$ at admission was significantly higher in non-survivors compared to survivors. Most of the patients (60.6\%) had a mild disease at the time of admission (Table 1).

$48.6 \%$ of the patients had lymphopenia $(<800 / \mu \mathrm{l})$ at admission, but neither the lymphopenia nor the lymphocyte count was significantly different between the survivor and non-survivor patients. Serum creatinine [198.0 $\mu \mathrm{mol} / \mathrm{l}$ (IQR: 97.2-293.7 $\mu \mathrm{mol} / \mathrm{l})$ vs. $132.6 \mu \mathrm{mol} / \mathrm{l}$ (IQR: $97.2-181.2 \mu \mathrm{mol} / \mathrm{l}$ ) respectively, $P=0.018$ ], CRP levels (during follow-up period, $>\times 10$ upper limit) were significantly higher $(71.4 \%$ vs. $38.9 \%, p=0.022)$ in nonsurvivors than survivors. Non-survivor patients had significantly higher (more than 2 times increase in the upper limit of normal) serum lactate dehydrogenase $(\mathrm{LDH})(61.5 \%$ vs. $23.1 \%, p=0.004)$ and aspartate transaminase (AST) levels (38.5\% vs. $11.8 \%, p=0.013$ ).

There was no statistically significant difference between the two groups in ferritin, hemoglobin, platelet, and serum albumin levels (Table 1).

Serum creatinine $[198.0 \mu \mathrm{mol} / \mathrm{l} \quad$ (IQR: $106.1-$ $265.2 \mu \mathrm{mol} / \mathrm{l}$ ) vs $132.6 \mu \mathrm{mol} / \mathrm{l}$ (IQR: $88.4-176.8 \mu \mathrm{mol} / \mathrm{l}$ ) vs. respectively, $P=0.016$ ], ferritin level [728 $\mu \mathrm{g} / \mathrm{l}$ (IQR $514.0-2000.0 \mu \mathrm{g} / \mathrm{l}$ ) vs $328 \mu \mathrm{g} / \mathrm{l}$ (IQR $129.0-814.0 \mu \mathrm{g} / \mathrm{l}$ ) respectively, $p=0.029$ ), CRP levels (during follow-up period, $>\times 10$ upper limit) $(72.7 \%$ vs. $35.6 \%$, respectively, $p=0.002$ ), LDH levels (during follow-up period, $>\times 2$ upper limit) $(76.2 \%$ vs $15.7 \%$, respectively, $p<0.001)$, AST levels (during follow-up period, $>\times 2$ upper limit) (38.1\% vs $9.1 \%$, respectively, $p=0.001$ ) were significantly higher in patients followed in the ICU than others. However, serum albumin levels were significantly lower [3.45 g/dl (IQR 2.9-3.8 g/dl) vs $3.8 \mathrm{~g} / \mathrm{dl}$ (IQR 3.5-4.1 g/dl), respectively, $p=0.003$ ] in patients followed in the ICU compared to the others. There was no statistically significant difference between the two groups in terms of hemoglobin, thrombocyte, and lymphocyte count at the time of admission (Table 2).

In terms of secondary outcome, serum creatinine levels [441.3 $\mu \mathrm{mol} / \mathrm{l} \quad$ (IQR: $262.5-735.5 \mu \mathrm{mol} / \mathrm{l}) \quad$ vs. $1.5 \mu \mathrm{mol} / \mathrm{l}$ (IQR: 89.7-177.7 $\mu \mathrm{mol} / \mathrm{l}$ ) respectively, $p=$ 0.05] serum ferritin levels [679 $\mu \mathrm{g} / \mathrm{l}$ (IQR:184-2260 mg/ dl) vs. $132.6 \mu \mathrm{g} / \mathrm{l}$ (IQR: $128-839 \mu \mathrm{g} / \mathrm{l}$ ) respectively, $p=$ 0.048], CRP levels (during follow up period, > $\times 10$ upper limit,73.9\% vs. $34.9 \%, p=0.001$ ) were significantly higher in patients who reached secondary outcome. Serum albumin level [3.4 g/dl (2.9-3.8) vs. $3.8 \mathrm{~g} / \mathrm{dl}(3.5-4.1), p=$ $0.002]$ and presence of lymphopenia rate $(<800 / \mu \mathrm{l})$ (90.9\% vs. $66.3 \% p=0.018)$ were significantly lower in these patients. Patients who reached the secondary outcome had significantly higher (more than 2 times increase in the upper limit of normal) serum LDH $(72.7 \%$ vs. $15.9 \%, p<0.001)$ and AST levels (36.4\% vs. $9.2 \%, \mathrm{p}<$ 0.001 ), and significantly higher (more than 10 times increase in the upper limit of normal) CRP (73.9\% vs. $34.9 \%, p=0.001)$. There was no statistically significant difference in terms of leucopenia, thrombocytopenia during hospitalization period between groups (Table 3). 
Table 3 Baseline characteristics, lab tests, medication, and follow-up parameters of the patients according to the secondary outcome (dead and/or ICU admission)

\begin{tabular}{|c|c|c|c|c|}
\hline \multirow[b]{2}{*}{ Characteristic } & & \multicolumn{2}{|c|}{$\begin{array}{l}\text { Secondary outcome } \\
\text { (dead and/or ICU admission) }\end{array}$} & \multirow[t]{2}{*}{$p$-value } \\
\hline & & $\begin{array}{l}\text { No } \\
N=86\end{array}$ & $\begin{array}{l}\text { Yes } \\
N=23\end{array}$ & \\
\hline \multicolumn{5}{|l|}{ Demographic information } \\
\hline Male Gender, n (\%) & & $52(60.5)$ & $12(52.2)$ & 0.276 \\
\hline Age (years), median (IQR) & & $48(38-56)$ & $55(44-64)$ & 0.085 \\
\hline \multirow[t]{2}{*}{ Donor type, $\mathrm{n},(\%)$} & Deceased & $11(12.8)$ & $6(26.1)$ & 0.118 \\
\hline & Living & $75(87.2)$ & $17(73.9)$ & \\
\hline$>60$ years $n, \%$ & & $12(13.9)$ & $9(39.1)$ & 0.004 \\
\hline Time from symptom onset to admission, days, median (IQR) & & $4.0(3.0-7.0)$ & $5.0(3.0-7.0)$ & $<0.001$ \\
\hline Transplantation duration, years, median (IQR) & & $5.0(3.5-9.25)$ & $6.0(3.0-9.5)$ & 0.545 \\
\hline Length of stay in hospital (days), median (IQR) & & $8.5(6.0-13.0)$ & $14.0(8.0-18.5)$ & $<0.001$ \\
\hline Tx Duration < 1 year $\mathrm{n}(\%)$ & & $13(15.1)$ & $4(17.4)$ & 0.789 \\
\hline \multirow[t]{7}{*}{ Coexisting disorder, n/N (\%) } & Diabetes mellitus & 18/84 (21.4) & $7 / 23(30.4)$ & 0.366 \\
\hline & Hypertension & $61(73.5)$ & $20(87.0)$ & 0.178 \\
\hline & Ischemic heart disease & 11/81 (13.6) & $7 / 22(31.8)$ & 0.046 \\
\hline & Heart failure & $1 / 81(1.2)$ & $3 / 23(13.0)$ & 0.009 \\
\hline & COPD & 3/83 (3.6) & $2 / 22(9.1)$ & 0.284 \\
\hline & Cancer & $6 / 83(7.2)$ & $0(0.0)$ & 0.194 \\
\hline & Chronic liver disease & 1/83 (1.2) & $0(0.0)$ & 0.605 \\
\hline \multirow[t]{9}{*}{ Cause of kidney disease, n (\%) } & Diabetic nephropathy & $9(10.5)$ & $4(17.4)$ & 0.231 \\
\hline & Glomerular disease & $8(9.3)$ & $5(21.7)$ & \\
\hline & Hypertensive nephrosclerosis & $22(25.6)$ & $6(26.1)$ & \\
\hline & ADPCKD & $4(4.7)$ & $1(4.3)$ & \\
\hline & Amyloidosis & $4(4.7)$ & $0(0.0)$ & \\
\hline & Chronic pyelonephritis & $6(7.0)$ & $0(0.0)$ & \\
\hline & Urological diseases & $6(7.0)$ & $0(0.0)$ & \\
\hline & Unknown & $25(29.1)$ & $6(26.1)$ & \\
\hline & Others & $2(2.3)$ & $0(0.0)$ & \\
\hline \multirow[t]{4}{*}{ Smoking, n (\%) } & Former smoked & $18(20.9)$ & $4(17.4)$ & 0.919 \\
\hline & Never smoker & $34(39.5)$ & $9(39.1)$ & \\
\hline & Current smoker & $1(1.2)$ & $0(0.0)$ & \\
\hline & Unknown & $33(38.4)$ & $10(43.5)$ & \\
\hline \multirow[t]{10}{*}{ Medications, n/N (\%) } & ACEi & 17/81 (21.0) & $4 / 22(18.2)$ & 0.772 \\
\hline & ARBs & $12 / 80(15.0)$ & $2 / 22(9.1)$ & 0.476 \\
\hline & Statins & 7/81 (8.6) & $4 / 20(20.0)$ & 0.144 \\
\hline & $\begin{array}{l}\text { Anticoagulant or antiplatelet } \\
\text { agent }\end{array}$ & $33 / 82(40.2)$ & $12 / 20(60.0)$ & 0.111 \\
\hline & Oral antidiabetics & $7 / 80(8.8)$ & $1 / 22(4.5)$ & 0.516 \\
\hline & Tacrolimus & $69(80.2)$ & $17(73.9)$ & 0.509 \\
\hline & CsA & $9(10.5)$ & $0(0.0)$ & 0.105 \\
\hline & MPA derivatives & $12(14.0)$ & $20(87.0)$ & 0.910 \\
\hline & MTORi & $9(10.5)$ & $3(13.0)$ & 0.726 \\
\hline & Azathioprine & $5 / 81(5.8)$ & $1 / 22(4.3)$ & 0.784 \\
\hline
\end{tabular}


Table 3 Baseline characteristics, lab tests, medication, and follow-up parameters of the patients according to the secondary outcome (dead and/or ICU admission) (Continued)

\begin{tabular}{|c|c|c|c|c|}
\hline \multirow[b]{2}{*}{ Characteristic } & & \multicolumn{2}{|c|}{$\begin{array}{l}\text { Secondary outcome } \\
\text { (dead and/or ICU admission) }\end{array}$} & \multirow[t]{2}{*}{$p$-value } \\
\hline & & $\begin{array}{l}\text { No } \\
N=86\end{array}$ & $\begin{array}{l}\text { Yes } \\
N=23\end{array}$ & \\
\hline & Prednisone & $84(97.7)$ & $22(95.7)$ & 0.599 \\
\hline Induction therapy, yes, n, (\%) & & $66(76.7)$ & $14(60.9)$ & 0.126 \\
\hline \multirow[t]{2}{*}{ Induction therapy n, (\%) } & ATLG & $56(65.1)$ & $11(47.8)$ & \multirow[t]{2}{*}{0.268} \\
\hline & Basiliximab & $10(11.6)$ & $3(13.0)$ & \\
\hline \multirow[t]{3}{*}{ Modification of immunosuppression n, (\%) } & No changed & $3(3.5)$ & $0(0.0)$ & \multirow[t]{3}{*}{0.076} \\
\hline & MPA/AZA withdrawal & $75(87.2)$ & $17(73.9)$ & \\
\hline & $\mathrm{CNI}+\mathrm{MPA} / \mathrm{AZA}$ withdrawal & $8(9.3)$ & $6(26.1)$ & \\
\hline \multirow{3}{*}{$\begin{array}{l}\text { COVID-19 related clinic presentation at the time of diagnosis, } n \\
\text { (\%) }\end{array}$} & Mild disease & $64(74.4)$ & $3(13.0)$ & \multirow[t]{3}{*}{$<0.001$} \\
\hline & Moderate Disease & $22(25.6)$ & $11(47.8)$ & \\
\hline & Severe-Critical Disease & $0(0.0)$ & $9(39.1)$ & \\
\hline \multirow[t]{8}{*}{ Presentation symptoms n, (\%) } & Fever & $54(62.8)$ & 16 (69.6) & 0.547 \\
\hline & Myalgia & $27(31.4)$ & $5(21.7)$ & 0.366 \\
\hline & Dyspnea & $35(40.7)$ & $18(78.3)$ & 0.001 \\
\hline & Diarrhea & $11(12.8)$ & $1(4.3)$ & 0.251 \\
\hline & Cough & 59 (68.6) & $13(56.5)$ & 0.277 \\
\hline & Throat pain & $4(4.7)$ & $2(8.7)$ & 0.450 \\
\hline & Headache & $11(12.8)$ & $3(13.0)$ & 0.974 \\
\hline & Fatigue & $35(40.7)$ & $10(43.5)$ & 0.324 \\
\hline \multirow[t]{9}{*}{ COVID-19 drug treatments, n/N (\%) } & Macrolides & $54 / 84(64.3)$ & $17 / 22(77.3)$ & 0.249 \\
\hline & Oseltamivir & $44(53.0)$ & $15 / 22(68.2)$ & 0.202 \\
\hline & Hydroxychloroquine & $85(98.8)$ & $23(100.0)$ & 0.603 \\
\hline & Lopinavir-ritonavir & $8 / 76(10.5)$ & 2/18 (11.1) & 0.942 \\
\hline & Favipiravir & $30 / 80(37.5)$ & 19/20 (95.0) & $<0.001$ \\
\hline & Glucocorticoids & $40 / 79(50.6)$ & 19/22 (86.4) & 0.003 \\
\hline & Tocilizumab & $4 / 79(5.1)$ & $6 / 20(30.0)$ & 0.001 \\
\hline & Anakinra & $3 / 76(3.8)$ & $0(0.0)$ & 0.365 \\
\hline & Apheresis / immunoadsorption & $0(0.0)$ & $3 / 21(14.3)$ & 0.001 \\
\hline \multirow[t]{7}{*}{ Laboratory findings at admission, median (IQR) } & Creatinine $(\mu \mathrm{mol} / \mathrm{l})$ & $\begin{array}{l}132.6(89.7- \\
177.7)\end{array}$ & $\begin{array}{l}441.3(262.5- \\
735.5)\end{array}$ & 0.050 \\
\hline & Albumin $(\mathrm{g} / \mathrm{dl})$ & $3.8(3.5-4.1)$ & $3.4(2.9-3.8)$ & 0.002 \\
\hline & Ferritin $(\mu \mathrm{g} / \mathrm{l})$ & $331(128-839)$ & $679(184-2260)$ & 0.048 \\
\hline & Hemoglobin $(\mathrm{g} / \mathrm{dl})$ mean \pm SD & $11.6 \pm 2.4$ & $11.6 \pm 2.1$ & 0.900 \\
\hline & Lymphocyte count $(/ \mu l)$ & $860(545-1385)$ & $700(460-950)$ & 0.018 \\
\hline & Lymphopenia $(<800 / \mu \mathrm{l})$ n, (\%) & $57(66.3)$ & $20(90.9)$ & 0.394 \\
\hline & Platelet count $\left(\times 10^{3} / \mu l\right)$ & $199(169-248)$ & $186(161-239)$ & 0.451 \\
\hline \multirow[t]{6}{*}{ Follow-up parameters, n (\%) } & Acute kidney injury, n (\%) & $31(36.0)$ & $15(65.2)$ & 0.012 \\
\hline & RRT, n (\%) & $0(0.0)$ & $4(17.4)$ & $<0.001$ \\
\hline & Bacterial superinfection, $\mathrm{n}(\%)$ & $6(7.0)$ & $3(13.0)$ & 0.348 \\
\hline & \multicolumn{4}{|c|}{ Laboratory tests during hospitalization, n (\%) } \\
\hline & Leucopenia $(<4.0 / \mu l)$ & $28(32.6)$ & $8(34.8)$ & 0.840 \\
\hline & Lymphopenia $(800 / \mu l)$ & $57(66.3)$ & $20(90.9)$ & 0.023 \\
\hline
\end{tabular}


Table 3 Baseline characteristics, lab tests, medication, and follow-up parameters of the patients according to the secondary outcome (dead and/or ICU admission) (Continued)

\begin{tabular}{|c|c|c|c|c|}
\hline \multirow[b]{2}{*}{ Characteristic } & & \multicolumn{2}{|c|}{$\begin{array}{l}\text { Secondary outcome } \\
\text { (dead and/or ICU admission) }\end{array}$} & \multirow[t]{2}{*}{$p$-value } \\
\hline & & $\begin{array}{l}\text { No } \\
N=86\end{array}$ & $\begin{array}{l}\text { Yes } \\
N=23\end{array}$ & \\
\hline & $\begin{array}{l}\text { Thrombocytopenia }\left(<150 \times 10^{3} /\right. \\
\mu l)\end{array}$ & $11(12.8)$ & $5(21.7)$ & 0.281 \\
\hline & LDH (> $2 \times$ upper limit of normal) & $13(15.9)$ & $16(72.7)$ & $<0.001$ \\
\hline & AST (> $2 \times$ upper limit of normal) & $7(9.2)$ & $8(36.4)$ & 0.002 \\
\hline & CRP (> $10 \times$ upper limit of normal) & $30(34.9)$ & $17(73.9)$ & 0.001 \\
\hline \multirow[t]{2}{*}{ The final situation, $n(\%)$} & Recover & $86(100.0)$ & $9(22.2)$ & $<0.001$ \\
\hline & Exitus & $0(0)$ & $14(77.8)$ & \\
\hline
\end{tabular}

p-values presented from the chi-square test, Fisher's exact test, t-test, or Mann-Whitney $\mathrm{U}$ test

Abbreviations: IQR interquartile range, $L O S$ length of stay in the hospital, COPD chronic obstructive pulmonary disease, $A D P C K D$ autosomal dominant polycystic kidney disease, CSA cyclosporine A, ACEi angiotensin-converting enzyme inhibitors, ARBs angiotensin II receptor blocker, mTORi mammalian target of rapamycin inhibitors, MPA mycophenolate derivatives, CNI calcineurin inhibitors, AZA azathioprine, ATLG anti-T lymphocyte globulin, RRT renal replacement therapy, CRP C reactive protein, $L D H$ lactate dehydrogenase, $A S T$ aspartate aminotransferase, $I C U$ intensive care unit

\section{Treatment of COVID-19}

Almost all patients received hydroxychloroquine (99.1\%), majority of the patients received macrolide (67\%), oseltamivir (56.2\%) glucorticoids (58.4\%) and favipiravir $(49.0 \%)$ while a smaller subset of the patients received tocilizumab (10.1\%) or anakinra (3\%) and lopinavir/ritonavir (10.6\%) (Table 1). There was significant difference in mortality among tocilizumab $(41.7 \%$ vs. $5.7 \%, \mathrm{p}<$ $0.001)$, glucocorticoids $(85.7 \%$ vs. $54 \%, p=0.026)$ and favipiravir $(91.7 \%$ vs. $43.2 \%, p=0.002)$ treatments of COVID-19.

\section{Predictors of primary and secondary outcomes}

In univariate analyzes, it was determined that the presence of ischemic heart disease, initial serum creatinine levels were associated with mortality. Both parameters were found to be predictive of mortality in multivariate analysis (Table 4).

In univariate analyzes, it was determined that older age ( $>60$ years), initial serum creatinine, ferritin, albumin levels, lymphocyte count were associated with ICU admission. However, older age and initial serum creatinine were found to be predictive in multivariate analysis (Table 5).

In univariate analyzes, age over 60 years, baseline lymphocyte counts, initial serum creatinine and albumin levels were found to be predictive of secondary outcome. In multivariate analysis, age over 60 years and initial lymphocyte count were found to be related to secondary outcome (Table 6).

\section{Discussion}

In our national registry, the mortality rate was $12.8 \%$ in KTx recipients hospitalized with COVID-19, unlike

Table 4 Univariate and multivariate logistic regression analysis of the parameters related to mortality

\begin{tabular}{|c|c|c|c|c|}
\hline & \multicolumn{2}{|l|}{ Univariate Analysis } & \multicolumn{2}{|l|}{ Multivariate Analysis } \\
\hline & Odds Ratio (95\% Cl) & $p$-value & Odds Ratio $(95 \% \mathrm{Cl})$ & $p$-value \\
\hline Age $>60$ years & $2.743(0.811-9.274)$ & 0.104 & & \\
\hline Male gender & $0.500(0.161-1.556)$ & 0.231 & & \\
\hline Presence of diabetes mellitus & $1.371(0.390-4.822)$ & 0.622 & & \\
\hline Presence of hypertension & $1.152(0.295-4.506)$ & 0.838 & & \\
\hline Presence of ischemic heart disease & $3.702(1.047-13.083)$ & 0.042 & $4.129(1.104-15.442)$ & 0.035 \\
\hline Initial lymphocyte count & $0.999(0.998-1.000)$ & 0.222 & & \\
\hline Initial serum ferritin level & $1.000(1.000-1.001)$ & 0.373 & & \\
\hline Initial serum albumin level & $0.492(0.178-1.360)$ & 0.172 & & \\
\hline Initial serum creatinine level & $1.520(1.016-2.274)$ & 0.042 & $1.681(1.083-2.608)$ & 0.021 \\
\hline
\end{tabular}


Table 5 Univariate and multivariate logistic regression analysis of the parameters related to the ICU admission

\begin{tabular}{|c|c|c|c|c|}
\hline & \multicolumn{2}{|l|}{ Univariate Analysis } & \multicolumn{2}{|l|}{ Multivariate Analysis } \\
\hline & Odds Ratio (95\% Cl) & $p$-value & Odds Ratio (95\% Cl) & $p$-value \\
\hline Age $>60$ years & $3.503(1.214-10.108)$ & 0.020 & $5.754(1.331-24.882)$ & 0.019 \\
\hline Male gender & $0.673(0.263-1.722)$ & 0.409 & & \\
\hline Presence of diabetes mellitus & $1.737(0.616-4.900)$ & 0.297 & & \\
\hline Presence of hypertension & $2.247(0.606-8.339)$ & 0.226 & & \\
\hline Presence of ischemic heart disease & $2.333(0.756-7.205)$ & 0.141 & & \\
\hline Initial lymphocyte count & $0.999(0.998-1.000) 0$ & 0.028 & $0.999(0.997-1.000)$ & 0.111 \\
\hline Initial serum ferritin level & $1.000(1.000-1.001)$ & 0.042 & $1.000(1.000-1.001)$ & 0.100 \\
\hline Initial serum albumin level & $0.281(0.111-0.714)$ & 0.008 & $0.864(0.249-2.996)$ & 0.818 \\
\hline Initial serum creatinine level & $1.747(1.142-2.674)$ & 0.010 & $1.757(1.016-3.036)$ & 0.044 \\
\hline
\end{tabular}

Abbreviations: $\mathrm{Cl}$ confidence interval

previous single-center reports that observed mortality rates of $24-30 \%$ [2, 16-18]. In multivariate analysis, age over 60 years, presence of ischemic heart disease, initial serum creatinine level and lymphocyte count were found to be predictors of disease severity and mortality.

COVID-19 mortality rates in the general population vary from center to center. In the first study of $191 \mathrm{pa}$ tients from China, mortality rates were found to be $28 \%$ [5]. In subsequent publications, these rates were reported to be $8 \%$ in New York, $14 \%$ in Italy, and $12 \%$ in Spain [1]. According to the national data of our Ministry of Health (about 2.464.030 COVID-19 patients) hospitalized until 30.01 .2021 , the overall mortality rate is $2.49 \%$ [19]. It is unclear whether the mortality in the kidney or any SOT recipients is higher than in the general inpatient population. COVID-19 mortality in SOT recipients is higher than the normal population and also varies between 18 and $30 \%$ in different centers [2, 7, 1618]. However, in a large cohort study evaluating patients hospitalized for COVID-19, mortality, need for ICU care, and mechanical ventilation support rates were similar between SOT recipients and non-transplant patients [20]. Another large study evaluating 482 SOT recipients with COVID-19 found that the overall mortality was similar to the general non-transplant patient population with similar comorbidities [21]. In a recent study evaluating KTx recipients with COVID-19, authors reported the AKI (52\%), requiring IMV (29\%) and overall mortality (32\%) [22]. Compared to our patient group, AKI and IMV rates seem to be lower in our cohort. KTx recipients had a number of comorbid conditions such as hypertension, diabetes, ischemic heart disease. Although diabetes mellitus was associated with severe disease in both the TANGO study [22] group and the French cohort [23], the presence of hypertension alone was not associated with death in both studies. Ischemic heart disease is common in KTx recipients and is the leading cause of mortality [24]. In our series, the rates of diabetes mellitus, hypertension, and ischemic heart disease were lower compared to other studies. In this study, a significant relationship was found between ischemic heart disease and mortality, but the presence of

Table 6 Univariate and multivariate logistic regression analysis of the parameters related to secondary outcome (dead and/or ICU admission)

\begin{tabular}{|c|c|c|c|c|}
\hline & \multicolumn{2}{|l|}{ Univariate Analysis } & \multicolumn{2}{|l|}{ Multivariate Analysis } \\
\hline & Odds Ratio (Cl 95\%) & $p$-value & Odds Ratio (Cl 95\%) & $p$-value \\
\hline Age $>60$ years & $3.964(1.407-11.171)$ & 0.009 & $4.123(1.152-14.753)$ & 0.029 \\
\hline Male gender & $1.668(0.661-4.209)$ & 0.278 & & \\
\hline Presence of diabetes mellitus & $1.604(0.573-4.492)$ & 0.368 & & \\
\hline Presence of ischemic heart disease & $2.404(0.650-8.891)$ & 0.189 & & \\
\hline Presence of hypertension & $2.404(0.650-8.891$ & 0.189 & & \\
\hline Initial lymphocyte count & $0.999(0.998-1.000)$ & 0.022 & $0.999(0.998-1.000)$ & 0.046 \\
\hline Initial serum ferritin level & $1.000(1.000-1.001)$ & 0.062 & & \\
\hline Initial serum albumin level & $0.275(0.109-0.694)$ & 0.006 & $0.638(0.216-1.885)$ & 0.416 \\
\hline Initial serum creatinine level & $1.630(1.086-2.446)$ & 0.018 & $1.573(0.989-2.502)$ & 0.056 \\
\hline
\end{tabular}


hypertension and diabetes mellitus was not found to be associated with mortality. Although the French cohort found an association between the presence of cardiovascular disease and severe illness and death, no similar findings were reported in the TANGO study. Since our patients were younger compared to patients in other cohorts, the adverse effects of diabetes mellitus may have been reduced, and therefore the significant relationship between diabetes and mortality observed in other studies may not have been detected in this study. The overall low mortality in our series can be explained by the fact that our patients were younger than the other cohorts and the disease was less severe due to the low frequency of comorbidities. On comparing our KTx recipients with the normal population, mortality was found higher in KTx recipients $(12.8 \%$ vs $2.49 \%)$ which is consistent with previous reports $[2,7,16-18]$.

According to the treatment algorithm of the Ministry of Health, the use of favipiravir was limited only to intensive care patients during the period of the study. Therefore, this situation with favipiravir was attributed to selection bias.

Lymphopenia is common in the course of COVID-19 in both the general population and SOT recipients, and several studies have shown an association between disease severity and lymphopenia [20, 21, 25-27]. Our findings are in line with previous reports.

Cytokine storm is an important situation in the course of COVID-19 patients and is associated with death [28, 29]. Steroids and tocilizumab are used as treatments for this condition [30-32]. In the RECOVERY study, which is a randomized clinical study, it was determined that the addition of $6 \mathrm{mg}$ dexamethasone to the usual treatment provided significant improvements in patients who needed oxygen or ventilator support [33]. Although some promising results have been reported in the early stages of the pandemic, later randomized trials revealed uncertainties regarding the efficacy of tocilizumab [31, 34]. In a recently published randomized placebocontrolled study in hospitalized patients with COVID-19 pneumonia, tocilizumab reduced the likelihood of mechanical ventilation or death to progress to the composite outcome, but no improvement in survival was found [35]. However, in a multicenter Spanish study evaluating 80 kidney transplant recipients with COVID-19, higher mortality was found in the group that received tocilizumab compared to those who did not [32]. We also did not observe any specific benefit from tocilizumab use.

However, it is difficult to interpret the negative results in patients receiving steroids and/or tocilizumab in our study. All patients were receiving steroids (methylprednisolone $60 \mathrm{mg} /$ day) and tocilizumab concurrently, so it is not possible to determine whether the net effect were associated with these medications. Also, our patients who received tocilizumab had more severe illnesses and a higher rate of oxygen needs. Because of this patient selection bias regarding tocilizumab use, we could not reach on conclusion about the relationship between tocilizumab use and negative outcomes.

In COVID-19, the presence of smoking and COPD was associated with severe disease and mortality [36]. However, no increased risk associated with smoking or COPD was reported in either the TANGO study [22] or the French cohort [23]. Similarly, we did not find an association between COPD and smoking and adverse clinical outcomes.

Although its frequency varies between centers [3739], AKI is common during the course of COVID-19 due to renal hypoperfusion, cytokine storm, and multiorgan failure. In our study, the frequency of AKI and RRT was 42.2 and $3.7 \%$, respectively. Both AKI and RRT were associated with disease severity and mortality. The significant relationship between mortality and creatinine levels at admission show that graft functions have prognostic significance in KTx recipients with COVID-19. These results are consistent with the TANGO study [22] and the French cohort [23].

Although this multicenter study has a large sample size, it has many limitations mainly due to its retrospective nature. For this reason, associations of some parameters with mortality reported may not reflect the causal relationship. Changes in treatment algorithms during the patient recruitment phase made it difficult to evaluate the results. Problems related to patient selection made it difficult to evaluate the treatment results, such as in the tocilizumab use. We also included PCR negative patients as clinical diagnosis of COVID-19. This issue should be considered on evaluating results.

In conclusion, COVID-19 in KTx has a high mortality rate, especially in patients with ischemic heart disease and poor graft function. Low lymphocyte counts at admission and age over 60 years increased the risk for their combined endpoint of death or ICU admission.

\footnotetext{
Abbreviations

KTx: Kidney transplantation; COVID-19: Coronavirus-19 disease; SOT: Solid organ transplantation; PCR: Polymerase chain reaction; IMV: Invasive mechanical ventilation; CT: computarized tomography; CNI: Calcineurin inhibitors; LOS: Length of stay; IQR: Interquartile range; LOS: Length of stay in hospital; COPD: Chronic obstructive pulmonary disease; ADPCKD: Autosomal dominant polycystic kidney disease; CsA: Cyclosporine A; ACEi: Angiotensinconverting enzyme inhibitors; ARBs: Angiotensin II receptor blocker; mTORi: Mammalian target of rapamycin inhibitors; MPA: Mycophenolate derivates; RRT: Renal replacement therapy; CRP: C reactive protein; LDH: Lactate dehydrogenase; AST: Aspartate aminotransferase; ICU: Intensive care unit; AKI: Acute kidney injury; Cl: Confidence intervals
}

Acknowledgments

Not applicable. 


\section{Authors' contributions}

Conception and design of the study (AY, KA, MA, ARO, SS, BT, HZT), data collection (All authors), statistical analysis (OAO, AY, SO), interpretation of the data (OAO, AY, SO), article writing $(\mathrm{OAO})$, manuscript review and modification (AY, SO). All authors read and approved the final version of the manuscript.

\section{Funding}

None.

\section{Availability of data and materials}

The datasets used and/or analyzed during the current study available from the corresponding author on reasonable request.

\section{Declarations}

\section{Ethics approval and consent to participate}

This study was approved by the University of Health Sciences, Istanbul Haseki Training and Research Hospital Ethics Committee with the number 41-2020. Informed consent from patients was waived due to the need for rapid data collection during the pandemic period by the institutional review board. The research complied with the Declaration of Helsinki [40].

\section{Consent for publication}

Not applicable.

\section{Competing interests}

The authors declare that they have no competing interests.

\section{Author details}

'Division of Nephrology, Department of Internal Medicine, Istanbul University Istanbul Faculty of Medicine, Istanbul, Turkey. ${ }^{2}$ Department of Nephrology, University of Health Sciences, Haseki Training and Research Hospital, Istanbul, Turkey. ${ }^{3}$ Division of Nephrology, Department of Internal Medicine, Mersin University Faculty of Medicine, Training and Research Hospital, Mersin, Turkey. ${ }^{4}$ Department of Nephrology, Hacettepe University Faculty of Medicine, Ankara, Turkey. ${ }^{5}$ Division of Nephrology, Memorial Hizmet Hospital Department of Internal Medicine, İstanbul, Turkey. ${ }^{6}$ Division of Nephrology, Department of Internal Medicine, Yeni Yuzyil University Faculty of Medicine, Istanbul, Turkey. ${ }^{7}$ Division of Nephrology, Department of Internal Medicine, Sakarya University Faculty of Medicine, Training and Research Hospital, Sakarya, Turkey. ${ }^{8}$ Department of Nephrology, University of Health Sciences, Haydarpasa Numune Training and Research Hospital, Istanbul, Turkey. ${ }^{9}$ Department of Transplantation, Koc University Hospital, Istanbul, Turkey. ${ }^{10}$ Division of Nephrology, Department of Internal Medicine, Istanbul University - Cerrahpasa Cerrahpasa Faculty of Medicine, Istanbul, Turkey. ${ }^{11}$ Division of Nephrology, Department of Internal Medicine, University of Health Sciences, Bagcilar Training and Research Hospital, Istanbul, Turkey. ${ }^{12}$ Department of Nephrology, University of Health Sciences, Izmir Bozyaka Training and Research Hospital, Izmir, Turkey. ${ }^{13}$ Department of Nephrology, Bursa City Hospital, Bursa, Turkey. ${ }^{14}$ Department of Nephrology, Darica Farabi Training and Research Hospital, Kocaeli, Turkey. ${ }^{15}$ Division of Nephrology, Department of Internal Medicine, Sakarya University Training and Research Hospital, Sakarya, Turkey. ${ }^{16}$ Şişli Memorial Hospital Department of Internal Medicine, Division of Nephrology, İstanbul, Turkey. ${ }^{17}$ Department of Nephrology, University of Health Sciences, Sultan 2, Abdulhamid Han Training and Research Hospital, Istanbul, Turkey. ${ }^{18}$ Department of Nephrology, Erciyes University Faculty of Medicine, Kayseri, Turkey. ${ }^{19}$ Department of Nephrology, Necmettin Erbakan University Meram Faculty of Medicine, Konya, Turkey. ${ }^{20}$ Division of Nephrology, Department of Internal Medicine, Atilim University Faculty of Medicine, Istanbul, Turkey. ${ }^{21}$ Division of Nephrology, Department of Internal Medicine, Ankara University Faculty of Medicine, Ankara, Turkey.

\section{Received: 5 October 2020 Accepted: 4 March 2021}

\section{Published online: 19 March 2021}

\section{References}

1. Aggarwal S, Garcia-Telles N, Aggarwal G, Lavie C, Lippi G, Henry BM. Clinical features, laboratory characteristics, and outcomes of patients hospitalized with coronavirus disease 2019 (COVID-19): early report from the United States. Diagnosis (Berl). 2020;7(2):91-6. https://doi.org/10.1515/dx-2020-0046.

2. Alberici F, Delbarba E, Manenti C, Econimo L, Valerio F, Pola A, Maffei C, Possenti S, Zambetti N, Moscato M, Venturini M, Affatato S, Gaggiotti M, Bossini N, Scolari F. A single center observational study of the clinical characteristics and short-term outcome of 20 kidney transplant patients admitted for SARS-CoV2 pneumonia. Kidney Int. 2020;97(6):1083-8. https:// doi.org/10.1016/j.kint.2020.04.002.

3. Wu Z, McGoogan JM. Characteristics of and important lessons from the coronavirus disease 2019 (COVID-19) outbreak in China: summary of a report of 72314 cases from the Chinese Center for Disease Control and Prevention. Jama. 2020;323(13):1239-42. https://doi.org/10.1001/jama.2020.2 648.

4. Onder G, Rezza G, Brusaferro S. Case-fatality rate and characteristics of patients dying in relation to COVID-19 in Italy. JAMA. 2020;323(18):1775-6. https://doi.org/10.1001/jama.2020.4683.

5. Zhou F, Yu T, Du R, Fan G, Liu Y, Liu Z, Xiang J, Wang Y, Song B, Gu X, et al, Clinical course and risk factors for mortality of adult inpatients with COVID19 in Wuhan, China: a retrospective cohort study. Lancet. 2020;395(10229): 1054-62. https://doi.org/10.1016/S0140-6736(20)30566-3.

6. Michaels MG, La Hoz RM, Danziger-Isakov L, Blumberg EA, Kumar D, Green M, Pruett TL, Wolfe CR. Coronavirus disease 2019: implications of emerging infections for transplantation. Am J Transplant. 2020;20(7):1768-72. https:// doi.org/10.1111/ajt.15832.

7. Nair V, Jandovitz N, Hirsch JS, Nair G, Abate M, Bhaskaran M, Grodstein E, Berlinrut I, Hirschwerk D, Cohen SL, Davidson KW, Dominello AJ, Osorio GA, Richardson S, Teperman LW, Molmenti EP. COVID-19 in kidney transplant recipients. Am J Transplant. 2020;20(7):1819-25. https://doi.org/10.1111/ajt.1 5967

8. Gandolfini I, Delsante M, Fiaccadori E, Zaza G, Manenti L, Degli Antoni A, Peruzzi L, Riella LV, Cravedi P, Maggiore U. COVID-19 in kidney transplant recipients. Am J Transplant. 2020;20(7):1941-3. https://doi.org/10.1111/ajt.1 5891.

9. Guillen E, Pineiro GJ, Revuelta I, Rodriguez D, Bodro M, Moreno A, Campistol JM, Diekmann F, Ventura-Aguiar P. Case report of COVID-19 in a kidney transplant recipient: does immunosuppression alter the clinical presentation? Am J Transplant. 2020;20(7):1875-8.

10. D'Antiga L. Coronaviruses and immunosuppressed patients: the facts during the third epidemic. Liver Transpl. 2020;26(6):832-4. https://doi.org/10.1002/ It.25756.

11. Aslam S, Mehra MR. COVID-19: yet another coronavirus challenge in transplantation. J Heart Lung Transplant. 2020;39(5):408-9. https://doi.org/1 0.1016/j.healun.2020.03.007.

12. Romanelli A, Mascolo S. Immunosuppression drug-related and clinical manifestation of coronavirus disease 2019: a therapeutical hypothesis. Am J Transplant. 2020;20(7):1947-8. https://doi.org/10.1111/ajt.15905.

13. European Centre for Disease Prevention and Control CdfcdC-, as of 29 May 2020. https://www.ecdc.europa.eu/en/covid-19/surveillance/case-definition. Accessed 29 Aug 2020.

14. Guidance to COVID-19 (SARS Cov2 Infection) (Scientific Board Study) Republic of Turkey Ministry of Health (published on April 14). Accessed 18 April.

15. Kdigo A: Work group. KDIGO clinical practice guideline for acute kidney injury. Kidney Int Suppl 2012, 2(1):1-138.

16. Pereira MR, Mohan S, Cohen DJ, Husain SA, Dube GK, Ratner LE, Arcasoy S, Aversa MM, Benvenuto LJ, Dadhania DM, Kapur S, Dove LM, Brown RS Jr, Rosenblatt RE, Samstein B, Uriel N, Farr MA, Satlin M, Small CB, Walsh TJ, Kodiyanplakkal RP, Miko BA, Aaron JG, Tsapepas DS, Emond JC, Verna EC. COVID-19 in solid organ transplant recipients: initial report from the US epicenter. Am J Transplant. 2020;20(7):1800-8. https://doi.org/10.1111/ajt.1 5941.

17. Akalin E, Azzi Y, Bartash R, Seethamraju H, Parides M, Hemmige V, Ross M, Forest S, Goldstein YD, Ajaimy M, Liriano-Ward L, Pynadath C, LoarteCampos P, Nandigam PB, Graham J, le M, Rocca J, Kinkhabwala M. Covid-19 and kidney transplantation. N Engl J Med. 2020;382(25):2475-7. https://doi. org/10.1056/NEJMc2011117.

18. Fernández-Ruiz M, Andrés A, Loinaz C, Delgado JF, López-Medrano F, San Juan R, González E, Polanco N, Folqueira MD, Lalueza A. COVID-19 in solid organ transplant recipients: a single-center case series from Spain. Am J Transplant. 2020;20(7):1849-58. https://doi.org/10.1111/ajt.15929. 
19. https://covid19.saglik.gov.tr/Eklenti/39230/0/covid-19-weekly-situa tionreport\%,2D\%2D-43pdf.pdf?_tag1=D3D202441F1F5165A33D16981E6544, EF7FCOA32F. In

20. Chaudhry ZS, Williams JD, Vahia A, Fadel R, Parraga Acosta T, Prashar R, Shrivastava P, Khoury N, Pinto Corrales J, Williams C, Nagai S, Abouljoud M, Samaniego-Picota M, Abreu-Lanfranco O, Busto R, Ramesh MS, Patel A, Alangaden GJ. Clinical characteristics and outcomes of COVID-19 in solid organ transplant recipients: a cohort study. Am J Transplant. 2020;20(11): 3051-60. https://doi.org/10.1111/ajt.16188.

21. Kates OS, Haydel BM, Florman SS, Rana MM, Chaudhry ZS, Ramesh MS, Safa K, Kotton CN, Blumberg EA, Besharatian BD, et al. COVID-19 in solid organ transplant: a multi-center cohort study. Clin Infect Dis. 2020:ciaa1097. https://doi.org/10.1093/cid/ciaa1097.

22. Cravedi P, Mothi SS, Azzi Y, Haverly M, Farouk SS, Perez-Saez MJ, RedondoPachon MD, Murphy B, Florman S, Cyrino LG, et al. COVID-19 and kidney transplantation: results from the TANGO international transplant consortium. Am J Transplant. 2020;20(11):3140-8. https://doi.org/10.1111/ajt.16185.

23. Caillard S, Anglicheau D, Matignon M, Durrbach A, Greze C, Frimat L, Thaunat O, Legris T, Moal V, Westeel PF. An initial report from the French SOT COVID registry suggests high mortality due to Covid-19 in recipients of kidney transplants. Kidney Int. 2020;98(6):1549-58. https://doi.org/10.1016/j. kint.2020.08.005.

24. Ojo AO, Hanson JA, Wolfe RA, Leichtman AB, Agodoa LY, Port FK. Longterm survival in renal transplant recipients with graft function. Kidney Int. 2000;57(1):307-13. https://doi.org/10.1046/j.1523-1755.2000.00816.x.

25. Ruan Q, Yang K, Wang W, Jiang L, Song J. Clinical predictors of mortality due to COVID-19 based on an analysis of data of 150 patients from Wuhan, China. Intensive Care Med. 2020;46(5):846-8. https://doi.org/10.1007/s00134020-05991-X.

26. Yang $X, Y u$ Y, Xu J, Shu H, Xia J, Liu H, Wu Y, Zhang L, Yu Z, Fang M: Clinical course and outcomes of critically ill patients with SARS-CoV-2 pneumonia in Wuhan, China: a single-centered, retrospective, observational study. Lancet Respir Med. 2020; 0 (0) [cited 2020 May 2]. In.; 2020.

27. Huang I, Pranata R. Lymphopenia in severe coronavirus disease-2019 (COVID-19): systematic review and meta-analysis. J Intensive Care. 2020;8:110.

28. Xu Z, Shi L, Wang Y, Zhang J, Huang L, Zhang C, Liu S, Zhao P, Liu H, Zhu L, Tai Y, Bai C, Gao T, Song J, Xia P, Dong J, Zhao J, Wang FS. Pathological findings of COVID-19 associated with acute respiratory distress syndrome. Lancet Respir Med. 2020;8(4):420-2. https://doi.org/10.1016/S2213-2600(20)3 0076-X.

29. Huang C, Wang $Y$, Li X, Ren L, Zhao J, Hu Y, Zhang L, Fan G, Xu J, Gu X, Cheng Z, Yu T, Xia J, Wei Y, Wu W, Xie X, Yin W, Li H, Liu M, Xiao Y, Gao H, Guo L, Xie J, Wang G, Jiang R, Gao Z, Jin Q, Wang J, Cao B. Clinical features of patients infected with 2019 novel coronavirus in Wuhan, China. Lancet. 2020;395(10223):497-506. https://doi.org/10.1016/S0140-6736(20)30183-5.

30. Di Giambenedetto S, Ciccullo A, Borghetti A, Gambassi G, Landi F, Visconti E, Zileri dal Verme L, Bernabei R, Tamburrini E, Cauda R: Off-label use of tocilizumab in patients with SARS-CoV-2 infection. J Med Virol 2020, 92(10): 1787-1788, DOI: https://doi.org/10.1002/jmv.25897.

31. Xu X, Han M, Li T, Sun W, Wang D, Fu B, Zhou Y, Zheng X, Yang Y, Li X, Zhang X, Pan A, Wei H. Effective treatment of severe COVID-19 patients with tocilizumab. Proc Natl Acad Sci U S A. 2020;117(20):10970-5. https:// doi.org/10.1073/pnas.2005615117.

32. Zhang C, Wu Z, Li J-W, Zhao H, Wang G-Q. Cytokine release syndrome in severe COVID-19: interleukin-6 receptor antagonist tocilizumab may be the key to reduce mortality. Int J Antimicrob Agents. 2020;55(5):105954. https:// doi.org/10.1016/j.jjantimicag.2020.105954.

33. Group RC. Dexamethasone in hospitalized patients with Covid19-preliminary report. N Engl J Med. 2020.

34. Luo P, Liu Y, Qiu L, Liu X, Liu D, Li J. Tocilizumab treatment in COVID-19: a single center experience. J Med Virol. 2020;92(7):814-8. https://doi.org/10.1 $002 / j m v .25801$.

35. Salama C, Han J, Yau L, Reiss WG, Kramer B, Neidhart JD, Criner GJ, KaplanLewis E, Baden R, Pandit L, Cameron ML, Garcia-Diaz J, Chávez V, MekebebReuter M, Lima de Menezes F, Shah R, González-Lara MF, Assman B, Freedman J, Mohan SV. Tocilizumab in patients hospitalized with Covid-19 pneumonia. N Engl J Med. 2021;384(1):20-30. https://doi.org/10.1056/ NEJMoa2030340.

36. Alqahtani JS, Oyelade T, Aldhahir AM, Alghamdi SM, Almehmadi M, Alqahtani AS, Quaderi S, Mandal S, Hurst JR. Prevalence, severity and mortality associated with COPD and smoking in patients with COVID-19: a rapid systematic review and meta-analysis. PLoS One. 2020;15(5):e0233147. https://doi.org/10.1371/journal.pone.0233147.

37. Cummings MJ, Baldwin MR, Abrams D, Jacobson SD, Meyer BJ, Balough EM, Aaron JG, Claassen J, Rabbani LE, Hastie J. Epidemiology, clinical course, and outcomes of critically ill adults with COVID-19 in New York City: a prospective cohort study. Lancet. 2020.

38. Hirsch JS, Ng JH, Ross DW, Sharma P, Shah HH, Barnett RL, Hazzan AD, Fishbane S, Jhaveri KD, Northwell C-RC, et al. Acute kidney injury in patients hospitalized with COVID-19. Kidney Int. 2020;98(1):209-18. https://doi.org/1 0.1016/j.kint.2020.05.006.

39. Richardson S, Hirsch JS, Narasimhan M, Crawford JM, McGinn T, Davidson KW, Barnaby DP, Becker LB, Chelico JD, Cohen SL. Presenting characteristics, comorbidities, and outcomes among 5700 patients hospitalized with COVID-19 in the New York City area. Jama. 2020;323(20):2052-9. https://doi. org/10.1001/jama.2020.6775.

40. Association WM: Declaration of Helsinki, ethical principles for medical research involving human subjects. 52 nd WMA General Assembly, Edinburgh, Scotland 2000.

\section{Publisher's Note}

Springer Nature remains neutral with regard to jurisdictional claims in published maps and institutional affiliations.
Ready to submit your research? Choose BMC and benefit from:

- fast, convenient online submission

- thorough peer review by experienced researchers in your field

- rapid publication on acceptance

- support for research data, including large and complex data types

- gold Open Access which fosters wider collaboration and increased citations

- maximum visibility for your research: over $100 \mathrm{M}$ website views per year

At BMC, research is always in progress.

Learn more biomedcentral.com/submissions 